\title{
CT-Guided Cervical Transforaminal Steroid Injections: Where Should the Needle Tip Be Located?
}

\author{
J.K. Hoang, D.P. Massoglia, M.A. Apostol, C.D. Lascola, J.D. Eastwood, and P.G. Kranz
}

\begin{abstract}
BACKGROUND AND PURPOSE: The aim of CT-guided CTSI is to inject medication into the foraminal region where the nerve root is inflamed. The optimal location for needle placement and therapeutic delivery, however, remain uncertain. The purpose of this study was to investigate how needle positioning and angle of approach impact the transforaminal distribution of injectate.
\end{abstract}

MATERIALS AND METHODS: We retrospectively reviewed fluoroscopic images from 90 CT-guided CTSI procedures for needle-tip location, needle angle, and contrast distribution. Needle-tip position was categorized as either foraminal zone, junctional, or extraforaminal. Distribution of contrast injected immediately before steroid administration was categorized as central epidural, intraforaminal, or extraforaminal in location. Needle-tip location and angle were correlated with contrast distribution.

RESULTS: The needle tip was most commonly placed in the junctional position ( 36 cases, $40 \%$ ), followed by foraminal ( 30 cases, $33 \%$ ) and extraforaminal (24 cases, 27\%) locations. Intraforaminal contrast distribution was highest when the needle location was foraminal (30/30, $100 \%$ ) or junctional $(35 / 36,97 \%)$, compared with extraforaminal $(7 / 24,29 \%)(P$ value $<.0001)$. There was no relationship between needle angle and contrast distribution.

CONCLUSIONS: Needle-tip location at the outer edge of the neural foramen (junctional location) correlated well with intraforaminal distribution of contrast for CT-guided CTSI and compared favorably with injectate distribution following foraminal zone needle positioning. Junctional needle positioning may be preferred over the foraminal zone by some proceduralists. Extraforaminal needle positioning resulted in less favorable contrast distribution, which may significantly diminish the therapeutic efficacy of CTSI.

ABBREVIATION: $C T S I=$ cervical transforaminal steroid injections

C ervical radiculopathy is a common debilitating condition that affects millions of people annually. ${ }^{1}$ CTSI with corticosteroids for pain management can be a successful treatment option when conservative measures fail and can reduce the need for surgical intervention. ${ }^{2}$ CTSI is guided by 2 main imaging modalities: conventional fluoroscopy and CT fluoroscopy. The use of CT fluoroscopic guidance has increased with technologic advances and greater availability. CT offers the advantage of better soft-tissue visualization for more precise needle placement. ${ }^{3}$

The aim of CTSI is to deliver steroids and/or local anesthetic

Received May 9. 2012; accepted after revision May 29.

From the Department of Radiology (J.K.H., C.D.L., J.D.E., P.G.K.), Division of Neuroradiology, and Department of Radiation Oncology (J.K.H.), Duke University Medical Center, Durham North Carolina; Department of Radiology (D.P.M.), Ralph Johnson VA Medical Center, Charleston, South Carolina; and Clinical Radiologists S.C. (M.A.A.), Springfield, Illinois.

Please address correspondence to Jenny K. Hoang, MD, Department of Radiology, Duke University Medical Center, Box 3808, Erwin Rd, Durham NC, 27710; e-mail: jennykh@gmail.com

http://dx.doi.org/10.3174/ajnr.A3266 medication into the perineural epidural compartment of the inflamed nerve root and close to the source of radiculitis, which may be the result of disk herniation or arthropathic stenosis. Authors differ regarding the ideal site of needle-tip placement, ${ }^{3-7}$ yet small but critical variations in needle positioning could significantly impact drug delivery and therapeutic efficacy. $\mathrm{Cho}^{4}$ describes a solely extraforaminal technique that does not use contrast material for CT-guided CTSI. Wolter et $\mathrm{al}^{5}$ describe positioning the patient prone rather than supine; and by using their dorsal approach, the needle tip can only be in the extraforaminal location. The techniques of Wagner ${ }^{6}$ and Cyteval et $\mathrm{al}^{7}$ for CT-guided CTSI use contrast, and the needle tip is located at the outer edge of the posterior foramen. These widely varying recommendations for needle positioning reveal uncertainty as to the optimal approach for therapeutic efficacy and are undoubtedly confusing for proceduralists learning to perform CTSI.

An ideal needle location should enable optimal delivery of medication to both the source of nerve root inflammation (eg, disk herniation, uncovertebral osteophyte) and the nerve root itself, but the location also balances therapeutic delivery with 

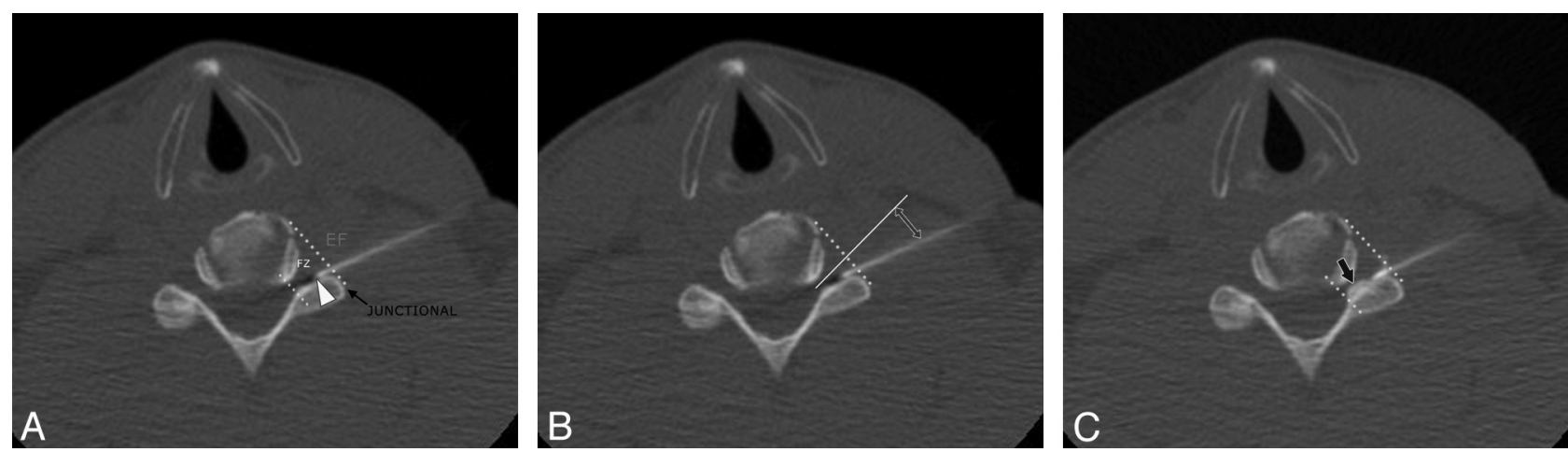

FIG 1. Classification of needle location and contrast distribution in a 42-year-old man with left-neck pain who had a CT-guided CTSI of the left C6 nerve root. A, CT fluoroscopic image shows the needle tip (arrowhead) in the foraminal zone (FZ). Foraminal zone and extraforaminal (EF) locations are separated by an oblique line from the anterolateral vertebral body to the lateral margin of the facet (junctional line). When the needle tip is on the oblique line, it is considered to be a junctional location (black arrow). B, Needle angle (double-headed arrow) is measured from the needle to a line (solid white line) perpendicular to the junctional line (dotted yellow line). C, CT fluoroscopic image of the cervical spine after the injection of dilute contrast. The contrast is seen in the intraforaminal region (black arrow).

safety to minimize complications. Although CTSI is safe in experienced hands, some proceduralists may be concerned about potential complications that could arise from needle placement deep within the foramen.

The aim of this study was to determine the impact of needle positioning and angle of approach on the distribution of injectate in the perineural intraforaminal space. We hypothesize that needle-tip location at the outer edge of the neural foramen (junctional) results in injectate distribution comparable with needle position inside the neural foramen (foraminal zone) and is a superior location compared with extraforaminal positioning for drug delivery.

\section{MATERIALS AND METHODS \\ Study Population}

We retrospectively reviewed 90 CT-guided CTSIs in 63 patients, performed from September 2009 to March 2010, that used contrast injection. Institutional review board approval was obtained for the retrospective review of previously obtained clinical and imaging data. The study was compliant with the Health Insurance Portability and Accountability Act.

Procedures were performed by 4 neuroradiologists, and the procedure was technically possible in all patients. There were 44 left and 46 right CTSIs in 36 female and 27 male subjects with a mean age 56 years. Injections were performed from the $\mathrm{C} 4$ to $\mathrm{C} 8$ nerve root level. The most commonly injected level was the C6 nerve root, which constituted 46 of 90 injections (51\%). None of the patients had complications during the injection or during an observation period after the injection.

\section{CT-Guided Injection Technique}

Our method for CT-guided CTSI has been previously described. ${ }^{3}$ Patients are positioned on the CT scanner gantry table (LightSpeed16, GE Healthcare, Milwaukee, Wisconsin) in the supine position with the head neutral or turned away from the side of the injection. A radiopaque skin marker is placed on the lateral neck, approximately at the plane of the earlobe. A preliminary unenhanced CT scan of the cervical spine is obtained through the area of interest, and the injection approach is planned. The pathway of the planned approach avoids the carotid space and the vertebral artery. Turning the head to the contralateral side can also help to position the carotid space medially and away from the path of the needle.

The skin is prepared and draped in a sterile fashion and is anesthetized with $1 \%$ lidocaine. With intermittent CT fluoroscopy, a 22-ga 3.5-inch needle is advanced into the skin and directed to the posterior aspect of the neural foramen, aiming for the needle tip to lie in or close to a foraminal zone location (Fig 1). A syringe containing dilute contrast $(2-\mathrm{mL}$ sterile saline and 1-mL iopamidol [Isovue M-200; Bristol Myers Squibb, Princeton, New Jersey]) is attached to the needle hub via a 31-inch microbore tubing (Microbore Extension Set; Cardinal Health, Dublin, Ohio). After negative aspiration, $0.2-0.5 \mathrm{~mL}$ of diluted contrast is slowly injected with the bevel of the needle directed ventrally. The purpose of contrast injection before steroid injection is to verify that the needle tip is not intravascular. A second role of contrast is to indicate the distribution that the subsequent steroid injection will follow. Dilute contrast was used because we have found the attenuation of undiluted contrast obscures the needle tip and makes repositioning the needle difficult.

If the contrast distribution is satisfactory, the contrast syringe is detached from the microbore tubing, and a syringe containing 1 $\mathrm{mL}$ of betamethasone $(3 \mathrm{mg} / \mathrm{mL})$ and $1 \mathrm{~mL}$ of bupivacaine $(2.5$ $\mathrm{mg} / \mathrm{mL}$ ) is connected. After initial heme-negative aspiration, the solution is slowly injected. We avoid using large-particle steroid suspensions with methylprednisolone and triamcinolone because these carry a risk of vessel embolization despite a negative aspirate for heme. ${ }^{8}$ Other interventionalists may favor dexamethasone solution, which is a nonparticulate steroid. Our choice is betamethasone because this is a low-particulate steroid that does not self-aggregate into large particles in solution, and it may have a longer duration of effect than nonparticulate steroids. ${ }^{9}$

\section{Image Analysis}

Two fellowship-trained neuroradiologists retrospectively evaluated CT fluoroscopic images from the CTSI procedures. Images were analyzed independently on a PACS workstation. A third proceduralist resolved disagreements. Readers classified the needletip location, measured the angle of needle approach, and classified 


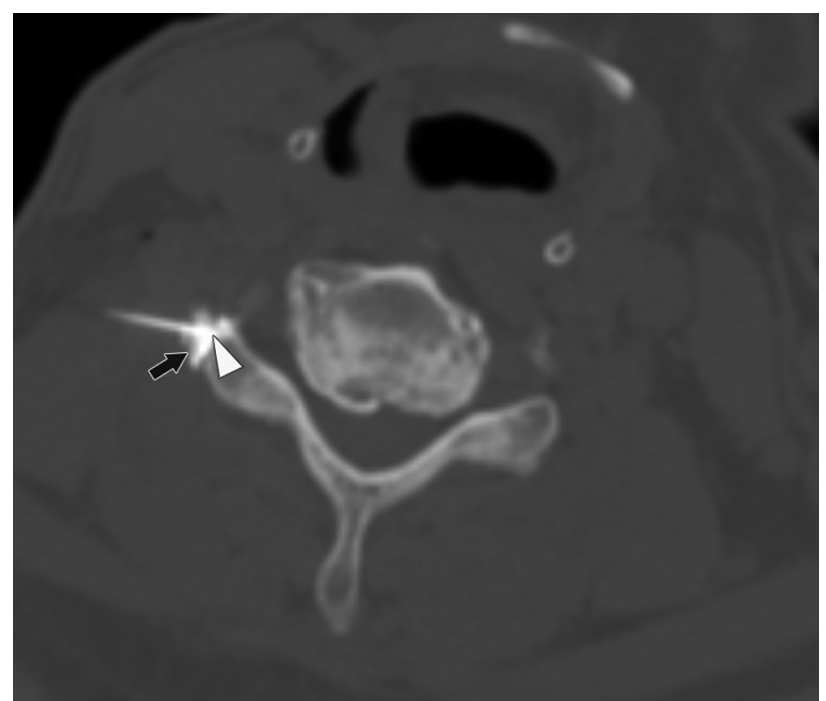

FIG 2. A 59-year-old man with right-neck pain radiating to the hand who had a CT-guided CTSI of the right C6 nerve root. The needle angle is $46^{\circ}$, and the tip of the needle is in the extraforaminal location (arrowhead) abutting the facet. The contrast is seen in the extraforaminal region (black arrow).

the contrast distribution. The needle-tip location was categorized as foraminal zone, junctional, and extraforaminal. These anatomic divisions are shown on Fig 1. Foraminal zone and extraforaminal locations were separated by an oblique line from the anterolateral vertebral body to the lateral margin of the facet. When the needle tip was on the oblique line (outer margin of the neural foramen), it was considered to be a junctional location. The needle angle was measured relative to the longitudinal axis of the neural foramen (Fig 1).

The distribution of contrast was classified as $\geq 1$ of the following: intraforaminal, extraforaminal, central epidural (intraspinal canal) regions, and "other" (Figs 1-3). "Other" regions included intravascular and subperiosteal contrast.

\section{Statistical Analysis}

The data were entered into an Excel spreadsheet (Microsoft, Bothell, Washington). Statistical analyses were performed by using SAS Enterprise (Version 4.2; SAS Institute, Cary, North Carolina). The Pearson $\chi^{2}$ test was used to determine the relationship between contrast distribution and needle-tip locations and level of injection. Correlation of contrast distribution with needle angle was evaluated with ANOVA. A 2-tailed $P$ value of $<.05$ was considered statistically significant.

\section{RESULTS}

\section{Needle Location and Contrast Distribution}

Table 1 shows the needle-tip location, needle angle, and contrast distribution by nerve root level for the 90 cases of CT-guided CTSI. The needle tip was most commonly placed in the junctional location ( 36 cases, $40 \%$ ), followed by the foraminal zone (30 cases, 33\%) and extraforaminal (24 cases, 27\%) location.

The distribution of contrast material generally lay in both the intraforaminal and extraforaminal locations. However, in 5 cases, there was an isolated intraforaminal distribution (Fig 1), and in 16 cases, there was an isolated extraforaminal distribution (Fig 2).

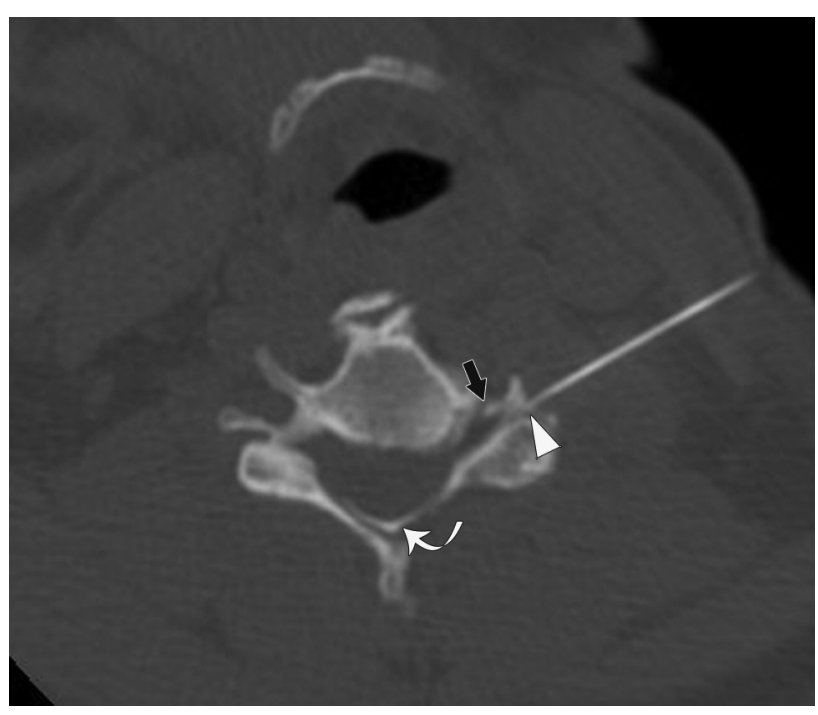

FIG 3. A 51-year-old woman with left-neck pain radiating to the forearm who had a CT-guided CTSI of the left C5 nerve root. The needle angle is $28^{\circ}$, and the tip of the needle is in the junctional location (arrowhead). Contrast is seen in the extraforaminal and intraforaminal (black arrow) regions. Contrast is also present in the central epidural space (curved arrow).

Central epidural distribution was seen in 12 cases in addition to intraforaminal contrast with or without extraforaminal contrast (Fig 3). Two cases had subperiosteal distribution of contrast, and in 1 case, there was intravascular contrast. The latter case was recognized at the time of injection, and medication was not injected.

\section{Correlation of Needle-Tip Location to Contrast Material Distribution}

Table 2 shows the relationship between needle-tip location and contrast distribution. Intraforaminal contrast material distribution was present in all cases when needle location was in the foraminal zone (30/30 cases, $100 \%)$ and was present in nearly all cases when needle location was junctional (35/36 cases, 97\%). When the needle tip was extraforaminal, intraforaminal distribution of contrast material was present in only 7 of 24 cases $(29 \%)(P$ value $<.0001)$. Central epidural contrast distribution was not present in any case of extraforaminal needle location but was present in 8 of 30 cases of foraminal zone (26\%) and 4 of 36 cases of junctional zone $(11 \%)$ needle locations $(P$ value $=.02)$.

\section{Needle Angle and Contrast Distribution}

The needle angle ranged from $-26^{\circ}$ to $+92^{\circ}$ with a mean of $34^{\circ} \pm$ $20^{\circ}$. The needle angle was shallower for lower cervical nerve root injections (Table 1).

The relationship between needle angle and contrast distributions was not statistically significant $(P$ value $>.10)$. However, there was a statistically significant relationship between injection level and angle ( $P$ value $=.002$ ), likely related to the different approach required in the lower cervical spine due to change in neck anatomy because higher level cervical foramina are angled more anteriorly than lower level cervical foramina. 
Table 1: Needle tip, mean angle, and contrast distribution by nerve root level ${ }^{\mathrm{a}}$

\begin{tabular}{|c|c|c|c|c|c|c|c|c|c|c|c|c|}
\hline \multirow{2}{*}{$\begin{array}{l}\text { Nerve } \\
\text { Root } \\
\text { Level }\end{array}$} & \multicolumn{3}{|c|}{ Needle-Tip Location } & \multicolumn{3}{|c|}{$\begin{array}{c}\text { Mean Needle Angle } \\
\text { for Needle-Tip Locations } \\
\text { and Level }\left({ }^{\circ}\right)\end{array}$} & \multicolumn{2}{|c|}{$\begin{array}{l}\text { Mean Needle Angle for all } \\
\text { Needle-Tip Locations }\left({ }^{\circ}\right)\end{array}$} & \multicolumn{4}{|c|}{ Contrast Distribution } \\
\hline & $\begin{array}{c}\text { Foraminal } \\
\text { Zone }\end{array}$ & Junctional & $\begin{array}{c}\text { Extra- } \\
\text { Foraminal }\end{array}$ & $\begin{array}{c}\text { Foraminal } \\
\text { Zone }\end{array}$ & Junctional & $\begin{array}{c}\text { Extra- } \\
\text { Foraminal }\end{array}$ & Mean & SD & $\begin{array}{c}\text { Intra- } \\
\text { Foraminal }\end{array}$ & $\begin{array}{c}\text { Extra- } \\
\text { Foraminal }\end{array}$ & $\begin{array}{r}\text { Central } \\
\text { Epidural }\end{array}$ & Other \\
\hline C4 & 1 & 3 & 1 & 32 & 50 & 23 & 41.1 & 16.4 & 4 & 5 & 2 & 0 \\
\hline C5 & 8 & 11 & 3 & 31 & 49 & 58 & 43.8 & 17.8 & 20 & 22 & 4 & 0 \\
\hline C6 & 17 & 13 & 16 & 28 & 39 & 33 & 32.7 & 19.1 & 34 & 42 & 6 & 3 \\
\hline C7 & 3 & 7 & 2 & 19 & 31 & 33 & 28.1 & 15.5 & 11 & 11 & 0 & 0 \\
\hline C8 & 1 & 2 & 2 & 18 & 6 & 5 & 7.6 & 22.6 & 3 & 3 & 0 & 0 \\
\hline Total (\%) & $30(34)$ & $36(39)$ & $24(27)$ & & & & & & $72(80)$ & $83(92)$ & $12(13)$ & $3(3)$ \\
\hline
\end{tabular}

${ }^{a}$ Contrast distribution could be seen in $>1$ site for each injection.

Table 2: Relationship between needle-tip location and contrast distribution

\begin{tabular}{|c|c|c|c|c|}
\hline & \multicolumn{3}{|c|}{ Needle-Tip Location } & \multirow[b]{2}{*}{$\begin{array}{c}P \\
\text { Value }\end{array}$} \\
\hline & $\begin{array}{c}\text { Foraminal } \\
\text { Zone }\end{array}$ & Junctional & $\begin{array}{c}\text { Extra- } \\
\text { Foraminal }\end{array}$ & \\
\hline \multicolumn{5}{|c|}{ Intraforaminal contrast } \\
\hline Present & 30 & 35 & 7 & $<.001$ \\
\hline Absent & 0 & 1 & 17 & \\
\hline \multicolumn{5}{|c|}{ Central epidural contrast } \\
\hline Present & 8 & 4 & 0 & .01 \\
\hline Absent & 22 & 32 & 24 & \\
\hline \multicolumn{5}{|c|}{ Extraforaminal contrast } \\
\hline Present & 27 & 34 & 22 & .79 \\
\hline Absent & 3 & 2 & 2 & \\
\hline
\end{tabular}

\section{Changing Needle Placement}

There were 14 cases (16\%) in which the needle was repositioned after initial injection of contrast. In 11 cases, this change occurred because the contrast distribution was predominantly in the extraforaminal region. In 2 cases, the needle was repositioned because of subperiosteal injection. One case was because the contrast was intravascular. The new needle locations after repositioning were in the foraminal zone in 5 patients and a junctional location in 5 patients. All of these 10 cases had new or increased intraforaminal contrast. In 4 cases, the needle tip remained extraforaminal because of severe degenerative changes and foraminal stenosis. In these cases, the contrast distribution remained predominantly extraforaminal.

The needle-tip position was not changed in 8 of 17 cases with extraforaminal needle-tip location and no intraforaminal contrast. It was difficult to determine the reason that the needle tip was not repositioned in all patients because this was a retrospective study. On evaluation of the images and reading the procedural report, the following were contributing factors: 1 ) difficult anatomy from a large osteophyte and severe foraminal stenosis, 2) patient discomfort due to proximity of the needle to the nerve root, and 3) proceduralist preference.

\section{DISCUSSION}

Several techniques for performing CT-guided CTSI have been reported. They vary in the description of patient position, needle technique, and use of contrast. ${ }^{3-7}$ Our study is the largest one to evaluate CT-guided CTSI and the first to explore needle position, to our knowledge. We did not have any reported complications and found that junctional and foraminal zone needle locations provided similar high rates of intraforaminal distribution of con- trast, significantly higher than that for the extraforaminal needle position.

Previous studies of the lumbar spine have reported that the ideal target site of a needle for transforaminal nerve root injection is the anterior epidural space between the inflamed nerve root and the posterior aspect of the herniated disk. ${ }^{10}$ There are no studies that evaluate the efficacy of cervical spine intraforaminal-versusextraforaminal medication distribution for pain relief, but we made the assumption that the mechanism of pain is similar in the lumbar and cervical spine, and thus intraforaminal medication delivery is favored over extraforaminal administration. Both injections may result in a short-term nerve block, but medium-tolong-term pain relief is likely significantly improved with intraforaminal administration of drugs closer to the source of nerve root inflammation. Our study found that optimal delivery may be observed with both junctional and foraminal zone needle positioning but is far less likely with an extraforaminal location. Another major finding is that the angle of needle approach does not correlate with intraforaminal contrast distribution. Instead, the angle of the needle is influenced by the injection level in the cervical spine. In the lower cervical spine, the angle is steeper with a more anterior approach because of the shoulders. In the upper cervical spine, the approach is more lateral with a larger angle because of the need to avoid the carotid sheath.

Anderberg et $\mathrm{al}^{11}$ also studied factors affecting the distribution of contrast for CTSI with CT imaging. Their method differed from ours in that patients had CTSI performed under conventional fluoroscopy followed by CT to visualize the contrast distribution after the needle was removed. They found that by CT criteria, only 5 of 9 injections were considered selective for the chosen nerve root and that the spread of contrast to other nerve roots and extraforaminal regions depended on the anatomy and size of the neural foramen and the volume of solution injected. By study design, these authors could not evaluate a third important factor affecting contrast distribution: location of needle tip at the time of injection. More precise assessment of the needle-tip location in the foraminal zone is possible with CT compared with conventional fluoroscopic guidance. We were also able to assess contrast distribution without delay and adjust the needle position accordingly. Additionally, the soft-tissue resolution of CT is also more advantageous for needle placement when the foraminal anatomy is abnormal because of the ability to visualize osteophytes or herniated disks.

The primary role of contrast for CTSI by conventional and CT fluoroscopy is to exclude an intravascular injection, which may 
lead to dissection or embolization of steroid particles. The vascular injection rate in studies using conventional fluoroscopic guidance was as high as $20 \%{ }^{8,12}$ There are no published data for the vascular injection rate with $\mathrm{CT}$ guidance, to our knowledge, but our study finds vascular injections to be uncommon with CT guidance because the carotid artery, vertebral artery, and jugular vein can be visualized on cross-sectional imaging and avoided. In contrast, conventional fluoroscopy only allows visualization of bony landmarks, which would explain a potentially higher intravascular injection rate. The single case of vascular injection in our study was in a tiny vessel that was $<1 \mathrm{~mm}$ in diameter and would have been difficult to see on conventional fluoroscopy. Another role of contrast is to confirm an optimal injection in the intraforaminal space. Sixteen percent of subjects had the needle repositioned after the injection of contrast. The new needle-tip location was deeper in the foraminal zone or junctional location rather than in the extraforaminal location. This positioning resulted in more than two-thirds of these cases having better intraforaminal distribution of contrast. The implication is that proceduralists who are tentative about placement of the needle deep in the foraminal zone could aim for the junctional location and reposition the needle deeper if they are not satisfied with the contrast distribution.

There are several limitations to our study. First, this is a retrospective study of a small number of patients at a single institution. Second, we are assuming that the spread of contrast accurately conveys the distribution of steroid medication. Although there are differences in the molecular weights and water solubilities of the 2 liquids, these parameters are unlikely to impact significantly their distribution within the perineural interstitium under convective delivery. Finally, we did not correlate needle positioning and injectate distribution with therapeutic outcome. Although it is possible that injections in the extraforaminal location may result in relatively good outcome due to the diffusion of the material into the neural foramina, we believe that therapeutic effects are maximized with direct delivery of drug to the source of nerve root inflammation in the neural foramina. Even if clinical outcome information was available, it may not be the best outcome measure for this study. An individual's pain score and change in score can be subject to many types of bias, may be multifactorial, and may not even be due to cervical nerve root impingement at the treated level. We used contrast distribution as the outcome in our study and think that this is an easily comparable and reasonable surrogate for technical success.

\section{CONCLUSIONS}

Needle-tip location in the outer edge of the neural foramen (junctional location) correlates well with intraforaminal distribution of contrast for CT-guided CTSI. This location may be preferred over the foraminal zone needle-tip placement by some proceduralists. Although some authors have previously reported that extraforaminal needle locations may be as effective as foraminal or junctional positioning for drug delivery, our results strongly suggest that extraforaminal positioning may significantly impair optimal administration of anti-inflammatory therapeutics.

\section{ACKNOWLEDGMENTS}

The authors thank Roxana Gafton, MD, for her editorial assistance.

\section{REFERENCES}

1. Radhakrishnan K, Litchy WJ, O'Fallon WM, et al. Epidemiology of cervical radiculopathy: a population-based study from Rochester, Minnesota, 1976 through 1990. Brain 1994;117(pt 2):325-35

2. Kolstad F, Leivseth G, Nygaard OP. Transforaminal steroid injections in the treatment of cervical radiculopathy. A prospective outcome study. Acta Neurochir (Wien) 2005;147:1065-70, discussion 1070

3. Hoang JK, Apostol MA, Kranz PG, et al. CT fluoroscopy-assisted cervical transforaminal steroid injection: tips, traps, and use of contrast material. AJR Am J Roentgenol 2010;195:888-94

4. Cho $\mathrm{CH}$. Cervical nerve injection: computed tomography guidance with intravenous contrast and extraforaminal needle placement. Series of seven consecutive case reports. Spine J 2010;10:e1-6

5. Wolter T, Mohadjer M, Berlis A, et al. Cervical CT-guided, selective nerve root blocks: improved safety by dorsal approach. AJNR Am J Neuroradiol 2009;30:336-37

6. Wagner AL. CT fluoroscopic-guided cervical nerve root blocks. AJNR Am J Neuroradiol 2005;26:43-44

7. Cyteval C, Thomas E, Decoux E, et al. Cervical radiculopathy: open study on percutaneous periradicular foraminal steroid infiltration performed under CT control in 30 patients. AJNR Am J Neuroradiol 2004;25:441-45

8. Malhotra G, Abbasi A, Rhee M. Complications of transforaminal cervical epidural steroid injections. Spine (Phila $\mathrm{Pa}$ 1976) 2009;34:731-39

9. Kushnerik V, Altman G, Gozenput P. Pharmacology of steroids used during epidural steroid injections. Techniques in Regional Anesthesia and Pain Management 2009;13:212-16

10. Botwin KP, Gruber RD, Bouchlas CG, et al. Fluoroscopically guided lumbar transforaminal epidural steroid injections in degenerative lumbar stenosis: an outcome study. Am J Phys Med Rehabil 2002;81:898-905

11. Anderberg L, Saveland H, Annertz M. Distribution patterns of transforaminal injections in the cervical spine evaluated by multi-slice computed tomography. Eur Spine J 2006;15:1465-71

12. Furman MB, Giovanniello MT, O'Brien EM. Incidence of intravascular penetration in transforaminal cervical epidural steroid injections. Spine (Phila Pa 1976) 2003;28:21-25 